\title{
RELACIÓN ENTRE AFECTO NEGATIVO Y DOMINIOS DE ESQUEMAS MALADAPTATIVOS TEMPRANOS COMO VARIABLES de personalidad en el Trastorno Depresivo Mayor: un ESTUDIO DE CASOS Y CONTROLES
}

\section{Relationship Between Negative Affect and Domains of Early Maladaptive Schemas as Personality Variables in the Major Depressive Disorder: A Case-Control Study}

\author{
Diana María Agudelo Vélez ${ }^{1}$, Yvonne Gómez-Maquet², \\ Catalina Uribe Castro $^{3}$, Ileana Manzanilla Africano ${ }^{4}$ \\ https://doi.org/10.17533/udea.rp.v12n2a03
}

\section{Resumen}

El presente estudio es de tipo empírico con un diseño cuasi-experimental de casos y controles cuyo propósito fue explorar el peso del afecto negativo (rasgo) y los dominios de los esquemas maladaptativos tempranos como indicadores de variables de personalidad asociados al trastorno depresivo. El grupo de casos estuvo conformado por 238 pacientes psiquiátricos con diagnóstico de Trastorno Depresivo Mayor y 236 personas de la comunidad general

Recibido: 2020-07-18 / Aceptado: 2020-10-11

Para citar este artículo en APA: Agudelo, D., Gómez-Maquet, Y. Uribe, C. y Manzanilla, I. (2020). Relación entre afecto negativo y dominios de esquemas maladaptativos tempranos como variables de personalidad en el trastorno depresivo mayor: un estudio de casos y controles. Revista de

Psicología de la Universidad de Antioquia, 12(2), e342946. doi: https://doi.org/10.17533/udea. rp.v12n2a03 sin este diagnóstico. Los pacientes presentaron niveles más altos de afecto negativo rasgo y mayores puntuaciones en los dominios de esquemas maladaptativos tempranos en comparación con los controles. El análisis de regresión logística mostró que la afectividad negativa explica un $59 \%$ y los dominios un $48 \%$ del trastorno depresivo. Estos resultados apoyan la dimensionalidad del modelo alternativo de clasificación de los trastornos de

Doctora en Psicología Clínica y de la Salud. Profesora, Departamento de Psicología, Universidad de los Andes. Correo: dm.agudelo932@ uniandes.edu.co; https://orcid.org/0000-00016658-449X.

2 Doctora en Psicología. Profesora, Departamento de Psicología, Universidad de los Andes; https://orcid.org/0000-0001-8629-3542

3 Magister en Psicología Clínica y de Salud. Psicóloga; https://orcid.org/0000-0003-0518-6687.

4 Magister en Psicología Clínica y de Salud. Psicóloga; https://orcid.org/0000-0003-3776-6109. 
personalidad del DSM-5, así como el mantenimiento del debate del trastorno depresivo de la personalidad.

\begin{abstract}
The current case-control study used a quasi-experimental design to explore the role that negative affectivity (as a trait) and the five domains of early maladaptive schemas have as personality variables related to depressive disorders. A secondary analysis of data was carried out in a case-control sample conformed by 238 psychiatric patients diagnosed with Major Depressive Disorder and 236 individuals without this diagnosis. Patients showed significantly greater levels of negative affectivity as a trait for both anxiety and depression, along with significantly higher scores in the five domains of early maladaptive
\end{abstract}

Palabras clave: afectividad negativa; esquemas maladaptativos tempranos; trastorno depresivo; trastorno de personalidad schemas compared to healthy individuals. A binary logistic regression analysis showed that a negative affectivity model explains 59\% of the variance of depression, while a model of early maladaptive schema domains explains $48 \%$. These findings favor the dimensional approach of the DSM-5 alternate classification model of personality disorders, as well as the possibility of reconsidering a depressive personality disorder diagnosis.

Keywords: negative affectivity, early maladaptive schemas, depressive disorder, personality disorders.

\section{Introducción}

El modelo alternativo de clasificación de los trastornos de personalidad en el DSM-5, parte de la introducción de la dimensionalidad como una forma de dar respuesta a las múltiples críticas frente al tratamiento categorial de los trastornos (Testal, Calderón y Garcelán, 2014). En este modelo, el trastorno se define en función de los impedimentos en el funcionamiento y la presencia de rasgos de personalidad patológicos (Asociación Americana de Psicología [APA], 2013). Las alteraciones en el funcionamiento se evidencian en los niveles del sí mismo (identidad y autodirección) y el nivel interpersonal (empatía e intimidad). Por su parte, los rasgos patológicos de la personalidad corresponden a las dimensiones de: afecto negativo, desapego, antagonismo, desinhibición y psicoticismo. Este modelo incluye los trastornos evitativo, antisocial, límite, esquizoide, narcisista y obsesivo compulsivo, pero adicionalmente considera la opción de trastorno de personalidad especificado por rasgos para dar cuenta de otros perfiles (antes presentes en el DSM-IV-TR), o formas nuevas que muestren rasgos de personalidad no incluidos en las clasificaciones actuales (APA, 2013). 
La alternativa de trastorno de personalidad especificado por rasgos contempla, igualmente, la afectación en el funcionamiento (niveles del sí mismo e interpersonal), pero, además, permite que se identifiquen rasgos patológicos en alguno(s) de los cinco dominios propuestos. Así, para el caso del presente artículo, se consideró lo relacionado con la afectividad negativa, que se refiere a la alta experiencia de emociones como el enojo, la ansiedad, la tristeza, la culpa, la preocupación, entre otras, así como las concomitantes expresiones comportamentales (aislamiento, autolesión, desmotivación, anhedonia, etc.). Lo anterior, como el paradigma que permitirá argumentar la existencia de un perfil de personalidad que podría recoger los síntomas de depresión persistente y un patrón común de afectividad negativa como ya otros autores lo habían planteado (Clark y Watson, 1991; 2003; Mineka, Watson y Clark, 1998). Por ejemplo, el Modelo de Clark y Watson denominado Modelo tripartito para la ansiedad y la depresión formula que los síntomas de la ansiedad y la depresión pueden agruparse en tres dominios: el afecto negativo, el afecto positivo y la activación fisiológica (Agudelo, Buela-Casal y Spielberger, 2007a; 2007b; Clark y Watson, 1991). Así, la depresión corresponde a un estado mixto de alto afecto negativo y bajo afecto positivo, mientras que la ansiedad se caracteriza por un estado de alto afecto negativo y alta activación fisiológica (Agudelo et al., 2007a; 2007b, Watson, Clark y Carey, 1988). De este modo, se determina que, aunque comparten un factor común, tienen unas características definitorias que permiten hacer una distinción entre ellos. Sin embargo, la comorbilidad evidenciada entre trastornos de ansiedad y depresión se explicaría, según el modelo, a partir del factor de alto afecto negativo que ambos comparten.

Otro modelo dimensional en psicopatología que trata de explicar la comorbilidad entre depresión y ansiedad es el de los factores supraordinados internalizante y externalizante (Achenbach y Edelbrock, 1978; Eaton, South y Krueger, 2010). El espectro internalizante incorpora una diversidad de síntomas inhibitorios o internos como la ansiedad, el miedo, la tristeza o depresión, la inhibición social y las quejas somáticas (Willner, Gatzke-Kopp y Bray, 2016). Es así como diferentes estudios han mostrado el solapamiento entre trastornos, tales como la distimia (ahora trastorno depresivo recurrente), el 
trastorno depresivo mayor (Klein, Schwartz, Rose y Leader, 2000) y trastornos de ansiedad como la ansiedad generalizada (Hidalgo y Sheehan, 2012), compartiendo un patrón de inestabilidad emocional propio de la dimensión internalizante (Eaton et al., 2010; Tyrer, Seivewright y Johnson, 2004; Willner et al., 2016).

Lo anterior permite avanzar en la línea de los rasgos de neuroticismo (altos niveles de afectividad negativa) como factor común en los trastornos del estado de ánimo, especialmente en el trastorno depresivo recurrente, lo cual apoyaría la idea de rasgos de personalidad que hacen más proclives a las personas a desarrollar este trastorno. A su vez, esta discusión remite al interés por diferenciar las características estado/rasgo, tal como lo han planteado diferentes autores (Agudelo et al., 2007a; 2007b; Ritterband y Spielberger, 1996; Spielberger, 1983; 1988; Spielberger, Jacobs, Russell y Crane, 1983; Spielberger et al., 2004; Spielberger et al., 1995; Spielberger, Ritterband, Reheiser y Brunner, 2003). Según esta aproximación, tanto en ansiedad como en depresión e ira, es posible identificar la presencia de características temporales, de reacción ante situaciones, que corresponderían a características/ estado; mientras que también es posible identificar características más estables/rasgos, que predisponen de cierta manera a la reactividad emocional y al desarrollo de ciertos trastornos y que, por lo tanto, estarían más asociadas a la personalidad.

\section{Relación entre depresión recurrente y personalidad depresiva}

El trastorno depresivo persistente es, como su precursor, la distimia, heterogéneo y con posibilidades de capturar diferentes condiciones depresivas y de ansiedad que tienen una clara relación con características de personalidad (Rhebergen y Graham, 2014).

La evidencia muestra una mayor cercanía del trastorno distímico (ahora depresión recurrente), con trastornos de la personalidad que con trastornos depresivos (Rhebergen et al., 2014). En apoyo a la idea anterior, un estudio reciente de Besenek (2020) encontró que en un grupo de niños y adolescen- 
tes con depresión o ansiedad, evaluados en consulta externa, y a quienes se les aplicó el cuestionario de personalidad DSM-5 Personality Inventory Child Form-Short Version (PID-5), no se encontraron diferencias estadísticamente significativas en el nivel de neuroticismo, aun controlando por los casos donde se identificaron comorbilidades, lo cual mostraría la existencia de un factor común entre estos dos grupos de trastornos.

Hay incluso evidencias desde la electrofisiología que muestran cómo, en jóvenes con altas puntuaciones en rasgos de personalidad depresiva, el procesamiento emocional es diferente a los participantes de un grupo control, en medidas de potenciales evocados, latencia de respuesta y recuerdo de contenido emocional negativo (Yu et al., 2019), lo cual podría estar marcando una ruta hacia el sesgo negativo como un rasgo estable en algunas personas que puede actuar como amplificador de los estados de ánimo negativos, aun en ausencia de depresión.

La discusión sobre el trastorno depresivo de la personalidad se ha centrado en evidencias a partir de las altas correlaciones entre el trastorno depresivo y la distimia con un patrón estable de personalidad, al que Huprich (2012) denominó trastorno depresivo de la personalidad. Este se caracteriza por un estilo de afecto negativo y perfeccionismo que, según los autores, está asociado con pérdidas tempranas en la infancia, autocrítica y negatividad (Huprich, 2012). En la misma línea, en un trabajo previo, Huprich (1998) había planteado que, desde etapas tempranas, los niños desarrollan un patrón perfeccionista como estrategia compensatoria para evitar el rechazo, el desamor y el abandono por parte de sus figuras significativas. En estudios como el de Huprich (2012), se encontró que la distimia, el trastorno depresivo mayor y el trastorno de personalidad depresivo, evaluado en dos muestras clínicas con el Depressive Personality Disorder Inventory, estuvieron altamente correlacionados y fueron predictivos entre sí. Además, se confirmó un patrón de alto perfeccionismo (evidenciado en un alto interés por evitar los errores, altos estándares personales, expectativas altas de los padres, crítica personal, dudas acerca de las acciones y un patrón generalizado de organización). Esta idea es cercana al planteamiento de Young, Klosko y Weisharr (2003) sobre los esquemas maladaptativos tempranos. 
Según Young et al. (2003), estos esquemas son patrones disfuncionales, estables y duraderos, compuestos por emociones, recuerdos, cogniciones y sensaciones corporales; patrones que se desarrollan durante la infancia y la adolescencia, y se relacionan con necesidades emocionales básicas no satisfechas, tales como el apego seguro, la autonomía, la libertad de expresión y el autocontrol, las cuales son necesarias para el desarrollo de una personalidad sana.

A partir de lo anterior, Young et al. (2003) identifican 18 esquemas maladaptativos tempranos (EMS) los cuales se agrupan en cinco dominios: desconexión y rechazo, perjuicio en autonomía y desempeño, límites inadecuados, tendencia hacia el otro y sobrevigilancia e inhibición. El primer dominio, desconexión y rechazo, se caracteriza por la incapacidad de los individuos para establecer vínculos seguros y satisfactorios con los demás, y se relaciona con la necesidad de seguridad, estabilidad, cuidado, empatía, expresión emocional y aceptación, que no fueron satisfechas. En el segundo dominio, perjuicio en autonomía y desempeño, se encuentran aquellas personas cuyas expectativas, sobre sí mismos y sobre su entorno, interfieren con su capacidad de funcionar de manera independiente y desempeñarse con éxito. En tercer lugar, se encuentra el dominio de límites inadecuados, en el cual los individuos tienen dificultades con la disciplina, el respeto por los derechos de los demás y el cumplimiento de promesas y objetivos a largo plazo. El cuarto dominio se denomina tendencia hacia el otro y comprende a individuos que sacrifican sus necesidades para suplir las de los demás y, de esta manera, obtener aprobación. Por último, se encuentra el dominio sobrevigilancia e inhibición, el cual agrupa las personas que suprimen sentimientos, siguen reglas estrictas o se autoimponen estándares sumamente rígidos, sacrificando la felicidad, la relajación, las relaciones cercanas y la salud.

En los últimos años se han realizado diferentes estudios que evidencian la asociación entre diversos esquemas maladaptativos tempranos y síntomas depresivos a lo largo del tiempo, y, además, que buscan identificar esquemas característicos para diferentes trastornos como ansiedad y depresión (Rafi, Adibsereshki y Aval, 2017; Alba y Calvete, 2019; Cámara y Calvete, 2012; Calvete, 2014; Davoodi et al., 2018; Halvorsen, Wang, Eisemann y Waterloo, 2010; Mateos-Pérez y Calvete, 2019; Nia, Sovani y Forooshani, 2014; Renner 
et al., 2012). Sin embargo, en la última década los estudios se han enfocado en el análisis por dominios de esquemas, más que en los esquemas individuales (Chen, Tam y Chang, 2019; Halvorsen et al., 2010; Mateos-Pérez y Calvete, 2019) y que en población clínica (Chen, Tam y Chang, 2019; Davoodi et al., 2018; Flink et al., 2019). En estos estudios se encuentra una relación entre los cinco dominios y el trastorno depresivo, siendo los dominios de desconexión y rechazo, perjuicio en autonomía y desempeño, sobrevigilancia e inhibición y límites inadecuados los más significativos.

En cuanto a los trastornos de personalidad, Hong y Tan (2020) condujeron un estudio con estudiantes universitarios para poner a prueba el modelo alternativo de los trastornos de personalidad del DSM-5, y encontraron que los individuos con tendencias patológicas de la personalidad (especialmente afectividad negativa y desapego) probablemente posean una amplia gama de vulnerabilidades cognitivas que pueden elevar su riesgo de bajo estado de ánimo, ansiedad y síntomas obsesivo-compulsivos.

La evidencia anterior parece mostrar una ruta hacia dos componentes que podrían apoyar lo descrito en el DSM-5 para el modelo alternativo de clasificación de los trastornos de personalidad, específicamente en lo relativo a una posible caracterización del trastorno depresivo de la personalidad, a partir de dos componentes diferenciales: la alta afectividad negativa y una vulnerabilidad cognitiva en función de los dominios de esquemas maladaptativos tempranos.

\section{Método}

\section{Diseño}

El presente estudio es de tipo cuasiexperimental con un diseño de casos y controles, basado en un análisis secundario de datos de la investigación: Estrés percibido y marcadores genéticos y epigenéticos en el trastorno depresivo mayor financiado por Minciencias. El objetivo fue explorar las características de afecto negativo (rasgo) y los dominios de los esquemas maladaptativos tempranos como indicadores de variables de personalidad, asociados con el trastorno depresivo. 


\section{Participantes}

La muestra estuvo compuesta por 474 personas divididas en dos grupos: muestra clínica (casos) y muestra no clínica (controles).

Los participantes de la muestra clínica $(n=238)$ fueron pacientes de dos clínicas psiquiátricas de la ciudad de Bogotá que tuvieron un diagnóstico de ingreso de trastorno depresivo mayor, basado en los criterios diagnósticos del CIE-10, el cual se corroboró a través de la Entrevista Estructurada Mini International Neuropsychiatric Interview (M.I.N.I).

La muestra no clínica $(n=236)$ estuvo conformada por personas de la comunidad general que no tuvieran un diagnóstico de trastorno depresivo mayor a partir de la entrevista M.I.N.I y apareados con la muestra clínica en características demográficas de edad y sexo. Esta muestra fue obtenida a través de varios medios: convocatoria abierta en dos instituciones universitarias y complementada con técnica de bola de nieve tanto en las universidades como con el personal administrativo de las instituciones psiquiátricas participantes. En la Tabla 1 se presenta la distribución de los grupos según variables sociodemográficas.

Tabla 1. Variables demográficas según grupo

\begin{tabular}{lccc}
\hline \multicolumn{1}{c}{ Variables } & $\begin{array}{c}\text { Casos } \\
\mathrm{N}(\%)\end{array}$ & $\begin{array}{c}\text { Controles } \\
\mathrm{N}(\%)\end{array}$ & $\begin{array}{c}\text { Diferencia entre } \\
\text { grupos }(\mathrm{p})\end{array}$ \\
\hline Mujeres & $161(67.65 \%)$ & $179(75.85 \%)$ & $0.32^{\mathrm{a}}$ \\
Hombres & $77(32.35 \%)$ & $57(24.15 \%)$ & $0.08^{\mathrm{a}}$ \\
Edad (años) & & & \\
$\quad$ Media & $33.9( \pm 10.31)$ & $34.49( \pm 12.97)$ & $0.58^{\mathrm{b}}$ \\
Min & 18 & 18 & \\
Max & 75 & 75 & \\
Estado civil & & & $0.69^{\mathrm{a}}$ \\
Soltero & $114(47.90 \%)$ & $120(50 \%)$ & $0.60^{\mathrm{a}}$ \\
Casado & $62(26.05 \%)$ & $68(28.33 \%)$ & $0.64^{\mathrm{a}}$ \\
Unión Libre & $38(15.97 \%)$ & $34(14.17 \%)$ & $0.32^{\mathrm{a}}$ \\
Separado & $20(8.40 \%)$ & $15(6.25 \%)$ &
\end{tabular}


Relación entre afecto negativo y dominios de esquemas maladaptativos tempranos como variables de personalidad en el Trastorno Depresivo Mayor

$\begin{array}{lccc}\text { Viudo } & 4(1.68 \%) & 2(0.83 \%) & 0.40^{\mathrm{a}} \\ \text { Datos perdidos } & 0(0 \%) & 1(0.42 \%) & \end{array}$

Nivel de escolaridad

$\begin{array}{lccc}\text { Primaria } & 17(7.14 \%) & 8(3.33 \%) & 0.07^{\mathrm{a}} \\ \text { Secundaria } & 87(36.55 \%) & 55(22.92 \%) & 0.007^{\text {*a }_{a}} \\ \text { Universitario } & 59(24.79 \%) & 87(36.25 \%) & 0.02^{*_{a}} \\ \text { Técnico } & 32(13.45 \%) & 37(15.42 \%) & 0.55^{\mathrm{a}} \\ \text { Posgrado } & 38(15.97 \%) & 52(21.67 \%) & 0.14^{\mathrm{a}} \\ \text { Datos Perdidos } & 5(2.10 \%) & 1(0.42 \%) & \end{array}$

Nota: ${ }^{*} \mathrm{p}<0.05^{* *} \mathrm{p}<0.005^{* * *} \mathrm{p}<0.001$; a. Prueba de Chi-cuadrado; b. Prueba T de Student.

\section{Instrumentos}

Entrevista Estructurada Mini International Neuropsychiatric Interview M.I.N.I (Sheehan et al., 1998). Traducción al español de Ferrando, Bobes, Gibert, Soto y Soto (2002): la M.I.N.I es una prueba diagnóstica estructurada que fue desarrollada por médicos psiquiatras y clínicos para los desórdenes psiquiátricos del DSM IV y el CIE-IO. Para este estudio, se consideró como Gold Standard que garantiza la evaluación de los criterios diagnósticos en la muestra y permite confirmar la presencia o no de trastornos mentales en los participantes.

Cuestionario de Depresión Estado-Rasgo IDER, adaptación española de Spielberger, Buela-Casal y Agudelo (2008): la prueba está compuesta por 20 ítems con dos escalas: estado y rasgo, cada una con 10 ítems. Se utilizó para evaluar el nivel actual de depresión (estado) y la predisposición de una persona a responder de manera depresiva (rasgo). Los niveles de confiabilidad de la prueba reportados por los autores oscilan entre 0.71 y 0.92 para las diferentes escalas en población general. En población colombiana se reportan alfas de Cronbach que oscilan entre 0.71 y 0.86 en población general (Agudelo, Gómez y López, 2014).

Inventario de Ansiedad Estado Rasgo STAI (Spielberger, Gorsuch y Lushene, 1970): el staI es una prueba de autoreporte de 40 ítems divididos en dos subescalas (estado y rasgo), cada una con 20 ítems. Fue utilizada para medir 
y diferenciar la ansiedad como estado y como rasgo, es decir, para medir la predisposición de una persona para responder de manera ansiosa y el nivel de ansiedad actual. La validación espańola desarrollada por Bermúdez (1978) reporta valores de consistencia interna para muestras, tanto normales como clínicas, que oscilan entre 0.82 y 0.92 .

Cuestionario de Esquemas de Young YSQ-SF (Londoño et al., 2012): cuestionario compuesto por 75 ítems que corresponden a 15 esquemas de los 5 dominios planteados por Jeffrey Young. Se utilizó para evaluar la presencia de esquemas maladaptativos tempranos en los participantes. Se estableció que este instrumento presenta propiedades psicométricas adecuadas para ser utilizado con población colombiana (Londoño et al., 2012).

\section{Procedimiento}

El estudio obtuvo el aval del comité de ética de las instituciones participantes y de un comité externo a una de las clínicas según la política de dicha institución. Los participantes firmaron un consentimiento informado y aceptaron que sus datos pudieran ser utilizados para futuros estudios. Para el caso de la muestra clínica los psiquiatras participantes identificaron los pacientes con diagnóstico de trastorno depresivo mayor y después de obtener su autorización, previa información del estudio, fueron contactados por un psicólogo clínico en formación adscrito al proyecto, quien llevó a cabo la entrevista diagnóstica M.I.N.I para confirmar el diagnóstico y el cumplimiento de los criterios de inclusión. Adicionalmente, aplicó la batería de cuestionarios.

Para el caso de la muestra no clínica se hizo una convocatoria mediante una conferencia pública de presentación del proyecto y posteriormente a través de un correo electrónico masivo en las instituciones educativas seleccionadas. De las personas que se presentaron se seleccionaron aquellas que tenían características similares en sexo y edad con la muestra clínica. Para completar la muestra no clínica se recurrió a la técnica de bola de nieve a través de los participantes ya seleccionados para este grupo. La evaluación se realizó según la disponibilidad de los participantes en su lugar de trabajo o estudio. El procedimiento de evaluación fue igual al seguido con la muestra clínica. Para garantizar el 
anonimato de los datos, se asignó un código único para el tratamiento de los datos de cada participante.

\section{Plan de análisis}

Una vez se comprobó que el grupo clínico y el control no se diferenciaban en las variables sexo y edad se calcularon los estadísticos descriptivos para las variables afecto negativo y esquemas maladaptativos tempranos. Se realizó una comparación de grupos para estas variables y se identificaron diferencias estadísticamente significativas a partir del estadístico no paramétrico $U$ de Mann Whitney. Finalmente, se realizó un análisis de regresión logística con el objetivo de evaluar el nivel de predicción de la afectividad negativa relacionada con depresión y ansiedad y el peso de los diferentes dominios de esquemas en el trastorno depresivo. Se evaluó el ajuste de los modelos a partir de los criterios de información Akaike (AIC) y Bayesiano (BIC) para seleccionar el modelo con mayor parsimonia. Todos los análisis se realizaron en RStudio (C) Versión 1.3.959 2009-2020 (RStudio Team, 2020).

\section{Resultados}

Antes de analizar los resultados relacionados con las variables del estudio es importante mencionar que un $55.8 \%$ de los pacientes cumplieron criterios diagnósticos tanto para trastorno depresivo mayor como para distimia (hoy denominada depresión recurrente). Sin embargo, esta proporción de pacientes con doble diagnóstico no es estadísticamente significativa frente al número de pacientes que solo obtuvo el diagnóstico de trastorno depresivo mayor. Ahora, al tomar como referencia a aquellos pacientes que presentaron altos niveles de afecto negativo rasgo para depresión y ansiedad, se evidencia que el 63\% de los pacientes con alta afectividad negativa cumplieron criterios diagnósticos también para distimia, siendo esta diferencia estadísticamente significativa $\left(\mathrm{x}^{2}\right.$ $=13.021 ; \mathrm{df}=1 ; \mathrm{p}<0.0003)$. Esto indicaría un alto nivel de cronicidad de la depresión. Es de anotar que en el grupo de controles solo un participante fue diagnosticado con distimia, por lo cual fue excluido de la muestra. 
En relación con la caracterización sociodemográfica de la muestra los datos reportados en la Tabla 1 muestran que la proporción de mujeres es mayor tanto para los casos como para los controles, aunque la diferencia no es estadísticamente significativa. Este dato será retomado en la discusión, pero refleja la evidencia epidemiológica sobre mayor presencia de depresión de las mujeres frente a los hombres. Para la variable edad se puede observar un amplio rango, sin embargo, la media se ubica en personas adultas jóvenes. Para la variable estado civil se encontró una distribución que podría estar relacionada con las características de edad ya descritas. Finalmente, la variable escolaridad presenta una diferencia entre casos y controles, siendo el nivel educativo más alto en el grupo de controles. Esto puede explicarse por las condiciones de recolección de este grupo. No obstante, aunque en esta característica los grupos no se encuentran apareados, los resultados no se ven afectados por esta condición.

A continuación, se reportan los resultados descriptivos y de comparación de grupos para las dos variables del estudio relacionadas con dimensiones de personalidad: afecto negativo (rasgo) y los esquemas maladaptativos tempranos agrupados por dominios. Los datos de la Tabla 2 muestran diferencias estadísticamente significativas entre pacientes y controles en ambas variables relacionadas con el afecto negativo rasgo. El grupo de pacientes obtuvo puntajes promedio superiores tanto para el rasgo de afecto negativo relacionado con depresión (IDER) como con ansiedad (STAI). El grupo control obtuvo puntajes que indican bajos niveles de afecto negativo rasgo en sus dos dimensiones. Esto podría confirmar la presencia de afectividad negativa como una característica más estable en el grupo de pacientes a diferencia del grupo control, lo cual se encuentra en línea con lo que este estudio espera mostrar. Si se revisan los resultados a nivel de estado hay una diferencia estadísticamente significativa, siendo los pacientes quienes reportan los valores más altos en estas medidas, como podría esperarse por su diagnóstico. 
Relación entre afecto negativo y dominios de esquemas maladaptativos tempranos como variables de personalidad en el Trastorno Depresivo Mayor

Tabla 2. Estadisticos descriptivos de los puntajes de la escala rasgo del IDER y STAI en función del grupo

\begin{tabular}{clllllll}
\hline \multirow{2}{*}{ Variable } & Grupo & Mínimo & Máximo & Media & Estándar & Mediana & U Mann Whitney \\
\hline \multirow{2}{*}{ IDER rasgo } & Casos & 10 & 40 & 28.3571 & 6.7382 & 29.5 & $3.465^{* * *}$ \\
& Controles & 10 & 28 & 15.4153 & 3.5366 & 15 & \\
\multirow{2}{*}{ IDER estado } & Casos & 10 & 40 & 27.5654 & 7.2428 & 29 & $5.169^{* * *}$ \\
& Controles & 10 & 30 & 15.5381 & 4.0469 & 15 & \\
\multirow{5}{*}{ STAI rasgo } & Casos & 4 & 58 & 38.384 & 10.597 & 40 & $3.782^{* * *}$ \\
& Controles & 0 & 46 & 17.1525 & 8.0743 & 16 & \\
\multirow{2}{*}{ STAI estado } & Casos & 0 & 60 & 32.3502 & 14.3214 & 35 & $7.356^{* * *}$ \\
& Controles & 0 & 48 & 12.1568 & 8.4605 & 11 & \\
\hline
\end{tabular}

Nota: ${ }^{*} \mathrm{p}<0.05^{* *} \mathrm{p}<0.005^{* * *} \mathrm{p}<0.001$

En la Tabla 3 se presentan los estadísticos descriptivos y la comparación de grupos para cada uno de los esquemas maladaptativos tempranos. Se encontró que los puntajes promedio y las medianas del grupo de pacientes son más altos para todos los esquemas en comparación con el grupo control, siendo esta diferencia estadísticamente significativa. Esto indicaría un nivel de compromiso cognitivo con presencia marcada de creencias, que podrían estar asociadas con el trastorno depresivo mayor.

Tabla 3. Estadisticos descriptivos de los esquemas maladaptativos tempranos según grupo

\begin{tabular}{llccccccc}
\hline & & \multicolumn{4}{c}{ Punto de } & \multicolumn{3}{c}{ Desviación U de Mann } \\
\multicolumn{1}{c}{ Esquema } & Grupo & Mínimo & Máximo & corte & Mediana & Media & Estándar & Whitney \\
\hline Deprivación & Casos & 5 & 30 & 15 & 16 & 16.1387 & 6.8364 & $10,144^{* * *}$ \\
emocional & Controles & 5 & 24 & 15 & 7 & 8.6624 & 4.325 & \\
Abandono & Casos & 5 & 30 & 20 & 23 & 21.6513 & 6.0713 & $6,902^{* * *}$ \\
& Controles & 5 & 29 & 20 & 10 & 11.3957 & 5.7312 & \\
Desconfianza y y & Casos & 5 & 30 & 17 & 18 & 18.1218 & 6.3384 & $10,226^{* * *}$ \\
abuso & Controles & 5 & 27 & 17 & 10 & 10.6979 & 4.9318 & \\
Defectuosidad & Casos & 5 & 30 & 12 & 16 & 16.3697 & 6.7134 & $8,153.5^{* * *}$ \\
& Controles & 5 & 23 & 12 & 7 & 8.2128 & 3.8271 & \\
Aislamiento & Casos & 5 & 30 & 17 & 18 & 18.5294 & 5.9019 & $9,068.5^{* * *}$ \\
social & Controles & 5 & 24 & 17 & 10 & 11.1795 & 4.3851 &
\end{tabular}



Ileana Manzanilla Africano

\begin{tabular}{llllccccc} 
Fracaso & Casos & 5 & 30 & 13 & 13.5 & 14.458 & 6.7808 & $12,330^{* * *}$ \\
& Controles & 5 & 24 & 13 & 7 & 8.3404 & 4.0556 & \\
Dependencia & Casos & 5 & 30 & 12 & 15 & 15.3655 & 6.0967 & $8,829^{* * *}$ \\
& Controles & 5 & 22 & 12 & 7 & 8.1064 & 3.6388 & \\
Vulnerabilidad & Casos & 5 & 30 & 18 & 18.5 & 18.542 & 5.9997 & $9,096.5^{* * *}$ \\
al dańo y enfer- & Controles & 5 & 28 & 18 & 9 & 10.5957 & 5.2618 & \\
medad & & & & & & & & \\
Apego & Casos & 5 & 30 & 15 & 14 & 14.5336 & 6.3748 & $10,853^{* * *}$ \\
& Controles & 5 & 23 & 15 & 7 & 8.1667 & 3.6807 & \\
Grandiosidad & Casos & 5 & 30 & 19 & 18 & 18.4093 & 5.3715 & $13,206^{* * *}$ \\
& Controles & 5 & 27 & 19 & 13 & 13.0596 & 5.1204 & \\
Autocontrol & Casos & 5 & 30 & 16 & 19 & 19.0882 & 5.5739 & $8,187^{* * *}$ \\
insuficiente & Controles & 5 & 25 & 16 & 11 & 11.265 & 4.7511 & \\
Subyugación & Casos & 5 & 30 & 13 & 18 & 17.6639 & 6.4491 & $8,097^{* * *}$ \\
& Controles & 5 & 25 & 13 & 9 & 9.4658 & 3.8637 & \\
Autosacrificio & Casos & 6 & 30 & 18 & 22 & 21.9118 & 5.4984 & $14,056^{* * *}$ \\
& Controles & 5 & 30 & 18 & 17 & 16.6538 & 5.7528 & \\
Estándares & Casos & 8 & 30 & 20 & 23 & 22.3571 & 4.8638 & $17,412^{* * *}$ \\
inflexibles & Controles & 5 & 30 & 20 & 19 & 19.0511 & 4.7373 & \\
Inhibición & Casos & 5 & 30 & 17 & 16.5 & 16.916 & 6.159 & $15,080^{* * *}$ \\
emocional & Controles & 5 & 28 & 17 & 11 & 11.766 & 5.8038 & \\
\hline
\end{tabular}

Nota: ${ }^{*} \mathrm{p}<0.05^{* *} \mathrm{p}<0.005^{* * *} \mathrm{p}<0.001$

Para efectos de este estudio, los esquemas se analizaron en su agrupación por dominios. Como se puede inferir de la Tabla 4, el grupo de pacientes se diferencia de manera significativa de los controles en todos los dominios.

Tabla 4. Estadisticos descriptivos de los dominios para el grupo control y la muestra clinica

\begin{tabular}{|c|c|c|c|c|c|c|c|}
\hline \multirow[b]{2}{*}{ Dominio } & \multirow[b]{2}{*}{ Grupo } & \multirow[b]{2}{*}{ Mínimo } & \multirow[b]{2}{*}{ Máximo } & \multicolumn{3}{|c|}{ Desviación } & \multirow{2}{*}{$\begin{array}{c}\text { U de Mann } \\
\text { Whitney }\end{array}$} \\
\hline & & & & Media & Estándar & Mediana & \\
\hline \multirow{2}{*}{$\begin{array}{l}\text { Desconexión y } \\
\text { rechazo }\end{array}$} & Casos & 6.6 & 30 & 18.1622 & 5.0381 & 18 & $5,420.5^{* * *}$ \\
\hline & Controles & 5 & 23.2 & 10.033 & 3.5294 & 9.2 & \\
\hline \multirow{2}{*}{$\begin{array}{l}\text { Perjuicio en } \\
\text { autonomía y } \\
\text { desempeńo }\end{array}$} & Casos & 5.25 & 26.25 & 15.7248 & 4.6017 & 15.5 & $6,205.5^{* * *}$ \\
\hline & Controles & 5 & 19.75 & 8.8053 & 3.0821 & 8 & \\
\hline \multirow{2}{*}{$\begin{array}{l}\text { Límites } \\
\text { Inadecuados }\end{array}$} & Casos & 7.5 & 29.5 & 18.7353 & 4.6288 & 19 & $8.680 .5^{* * *}$ \\
\hline & Controles & 5 & 23 & 12.1723 & 4.2966 & 12 & \\
\hline
\end{tabular}


Relación entre afecto negativo y dominios de esquemas maladaptativos tempranos como variables de personalidad en el Trastorno Depresivo Mayor

\begin{tabular}{llcccccc}
\hline $\begin{array}{llc}\text { Tendencia hacia } \\
\text { el otro }\end{array}$ & Casos & 7 & 30 & 19.7878 & 4.9572 & 19.5 & $8,262^{* * *}$ \\
\cline { 1 - 4 } Sobrevigilancia e e & Controles & 5 & 26.5 & 13.0809 & 4.033 & 13 & \\
inhibición & 7.5 & 30 & 19.6366 & 4.307 & 19.5 & $13,586^{* * *}$ \\
& Controles & 5.5 & 26.5 & 15.4085 & 4.1468 & 15.5 & \\
\hline
\end{tabular}

Nota: ${ }^{*} \mathrm{p}<0.05^{* *} \mathrm{p}<0.005^{* * *} \mathrm{p}<0.001$.

Para establecer el nivel de predicción de las dos variables de dimensiones de personalidad, afecto negativo y dominios de esquemas maladaptativos tempranos para el trastorno depresivo, se llevó a cabo un análisis de regresión logística binaria evaluando varios modelos y seleccionando el más parsimonioso. Todos los modelos mostraron ser estadísticamente significativos en la predicción del trastorno depresivo $(\mathrm{p}<0.001)$ y se tomaron en cuenta el criterio de información de Akaike (AIC) y el criterio de información Bayesiano (BIC) para evaluar la calidad relativa de los modelos.

Inicialmente, se había planteado la posibilidad de evaluar un modelo que incluyera tanto los dominios de esquemas maladaptativos como el rasgo de afecto negativo (medido a través de las escalas de IDER rasgo y STAI rasgo). Sin embargo, al evaluar la multicolinealidad entre estas variables, se evidenciaron altos coeficientes de correlación entre afecto negativo y los dominios de esquemas maladaptativos; por esta razón, no se hace viable su inclusión en un único modelo, se calcularon modelos separados para cada grupo de variables.

Para la variable de dominios de esquemas en el Modelo 1.1 se incluyeron los cinco dominios de esquemas para establecer su nivel de predicción, siendo el dominio de Sobrevigilancia e Inhibición el único que no aporta a la varianza explicada del trastorno depresivo (ver Tabla 5). Por lo tanto, se planteó un Modelo 1.2 sin incluir este dominio, lo cual se justifica por una reducción en el AIC y BIC, así como un mantenimiento en el nivel de explicación de la varianza del trastorno depresivo al presentar un $\mathrm{R}^{2}$ de McFadden de 0.48 . Así, este segundo modelo evidencia que el aumento en una unidad en el puntaje de los dominios de Desconexión y rechazo, Perjuicio en autonomía y desempeño, Limites inadecuados y Tendencia hacia el otro incrementa 1.19, $1.16,1.12$ y 1.11 veces la probabilidad de padecer un trastorno depresivo, respectivamente. 
Por su parte, en la Tabla 6 se encuentran los resultados de la regresión logística del rasgo afecto negativo, medido a través del STAI/R y el IDER/R. Estos indican la aceptación del modelo, en el cual tanto el rasgo relacionado con depresión como con ansiedad predicen mejor el trastorno depresivo, obteniendo un $\mathrm{R}^{2}$ de McFadden de 0.59.

Tabla 5. Modelo de predicción del trastorno depresivo en función de los dominios de esquemas maladaptativos tempranos

\begin{tabular}{|c|c|c|c|}
\hline Variable predictora & Estadísticos & Modelo 1.1 & Modelo 1.2 \\
\hline \multirow[t]{2}{*}{ Desconexión y Rechazo } & OR & $1.1884^{* * *}$ & $1.1884^{* * *}$ \\
\hline & IC $95 \%$ & $1.081-1.3132$ & $1.0874-1.3059$ \\
\hline \multirow{2}{*}{$\begin{array}{l}\text { Perjuicio en Autonomía y } \\
\text { Desempeño }\end{array}$} & OR & $1.1612^{* *}$ & $1.1611^{* *}$ \\
\hline & IC $95 \%$ & $1.0525-1.2842$ & $1.0531-1.2836$ \\
\hline \multirow[t]{2}{*}{ Límites Inadecuados } & $\mathrm{OR}$ & $1.1171^{* *}$ & $1.1171^{* *}$ \\
\hline & IC $95 \%$ & $1.0361-1.2067$ & $1.0373-1.2055$ \\
\hline \multirow[t]{2}{*}{ Tendencia hacia el Otro } & OR & $1.1095^{*}$ & $1.1095^{*}$ \\
\hline & IC $95 \%$ & $1.0266-1.2$ & $1.0267-1.2009$ \\
\hline \multirow{9}{*}{$\begin{array}{l}\text { Sobrevigilancia e } \\
\text { Inhibición }\end{array}$} & OR & 0.9999 & \\
\hline & IC $95 \%$ & $0.2414-1.0812$ & \\
\hline & Intercepto (OR) & $0.0005^{* * *}$ & $0.0005^{* * *}$ \\
\hline & Observaciones & 472 & 472 \\
\hline & Ajuste Modelo & $\chi^{2}(5)=319.199^{* * *}$ & $\chi^{2}(4)=319.199^{* * *}$ \\
\hline & Log Likelihood & -168.250 & -168.250 \\
\hline & R2 (McFadden) & 0.488 & 0.488 \\
\hline & AIC & 348.499 & 346.499 \\
\hline & BIC & 373.454 & 367.295 \\
\hline
\end{tabular}

Nota: ${ }^{*} \mathrm{p}<0.05^{* *} \mathrm{p}<0.005^{* * *} \mathrm{p}<0.001$ 
Relación entre afecto negativo y dominios de esquemas maladaptativos tempranos como variables de personalidad en el Trastorno Depresivo Mayor

Tabla 6. Modelos de predicción del trastorno depresivo en función del afecto negativo

\begin{tabular}{lll}
\hline Variable & Estadísticos & Modelo \\
\hline IDER Rasgo & OR & $1.2984^{* * *}$ \\
& IC $95 \%$ & $1.1927-1.4249$ \\
STAI Rasgo & OR & $1.0864^{* *}$ \\
& IC $95 \%$ & $1.0386-1.1392$ \\
& Intercept (oR) & $0.0003^{* * *}$ \\
Observaciones & 472 \\
& Ajuste Modelo & $X^{2}(2)=387.689^{* * *}$ \\
& Log Likelihood & -134.013 \\
R2 (McFadden) & 0.592 \\
AIC & 274.026 \\
BIC & 286.503 \\
\hline
\end{tabular}

Nota: ${ }^{*} \mathrm{p}<0.05^{* *} \mathrm{p}<0.005^{* * *} \mathrm{p}<0.001$

\section{Discusión}

El objetivo del presente estudio fue explorar el afecto negativo (rasgo) y los dominios de los Esquemas Maladaptativos Tempranos como indicadores de variables de personalidad asociados al trastorno depresivo. Es importante tener presente que no se utilizó ningún inventario de personalidad. Las inferencias sobre este constructo se hicieron a partir de la identificación de aproximaciones, como serían las mediciones de afecto negativo/rasgo (en cuanto características estables y duraderas del afecto negativo) y de los esquemas y dominios (entendidos, desde la perspectiva teórica como un sistema de pensamiento estable). Si bien la literatura podría soportar esta inferencia, es claro que puede constituir también una limitación del estudio, al no usarse una medida validada de personalidad.

Asimismo, es importante hacer notar que, de la muestra clínica, un porcentaje notorio $(55.8 \%)$ cumplió criterios diagnósticos para distimia. Este dato es relevante dado el interés del estudio frente a la idea de apoyo a un perfil de personalidad depresónico y soportaría la evidencia encontrada por Huprich 
y colaboradores a lo largo de sus estudios, en relación con la alta comorbilidad e incluso el valor predictivo entre trastornos del estado de ánimo (Huprich, 1998, 2012, 2013), así como la fuerte asociación con rasgos de personalidad según lo describen Rhebergen y Graham (2014). Pero más llamativo es cómo al comparar los resultados con punto de corte alto para afectividad negativa/ rasgo en la muestra clínica, los datos indican que los pacientes con distimia tuvieron las puntuaciones mayores, siendo esta diferencia estadísticamente significativa. Estos datos son consistentes con lo reportado por autores como Achenbach et al. (1978), Besenek (2020) y Eaton et al. (2010) sobre la alta comorbilidad entre ansiedad y depresión y el elemento compartido en términos de alta afectividad negativa.

En relación con las características sociodemográficas se destaca que el presente estudio analizó de manera preferente la edad y el sexo dado que la literatura señala diferencias importantes en la presentación de los síntomas y del diagnóstico en general de los trastornos del estado de ánimo según estas dos variables, así se evidencia que las mujeres reportan mayores niveles de depresión que los hombres, consultan más y los profesionales de la salud son más proclives a diagnosticar este cuadro en ellas que en los hombres (Agudelo et al., 2007a; Londońo y González-Rodríguez, 2016). A su vez, la edad se ha asociado con diferencia en la presentación de síntomas en los trastornos del estado de ánimo entre niños, adolescentes, adultos y adultos mayores (Gotlib y Hammen, 2015). Por esta razón este estudio se concentró en una muestra de adultos que pudieran compartir características similares frente al trastorno.

Los resultados mostrados en la Tabla 2 evidencian, tal como se mostró en estudios previos (Ritterband et al., 1996; Spielberger, 1983, 1988; Spielberger et al., 1983; Spielberger et al.; 1995; Spielberger et al., 2003; Spielberger et al., 2004;), que hay diferencia en la muestra clínica, no solo en la medida de estado (que describiría la presencia del trastorno depresivo con el cual los participantes ingresaron al estudio), sino, y de manera más contundente, una diferencia estadísticamente significativa en la medición de afecto negativo/ rasgo, mostrando el carácter dimensional del trastorno depresivo como lo describieran Agudelo et al. (2007a; 2007b); y, en coherencia con lo señalado en el modelo del DSM-5 para los trastornos de personalidad, respecto de las 
características de afectividad negativa. Asimismo, se puede evidenciar el factor de afecto negativo descrito por Clark et al., $(1991,2003)$ y Mineka et al., (1998). En todo caso, un posterior análisis de la comorbilidad presentada por los sujetos del presente estudio podría aportar evidencia aún más fuerte en la línea que aquí se describe.

Por su parte, los resultados en relación con los esquemas maladaptativos tempranos que se reportan en las tablas 3 y 4 muestran también soporte a los planteamientos de Young et al. (2003) acerca de la relación que tienen los esquemas con el desarrollo de diferentes trastornos, entre ellos la depresión. En el presente estudio se encontró que la muestra clínica presentó mayores puntuaciones (todas con diferencias significativas) en todos los esquemas evaluados, al igual que en relación con los dominios (excepto para el dominio de sobrevigilancia e inhibición) respecto de la muestra no clínica. Llama la atención lo observado, por ejemplo, con el esquema de estándares inflexibles, cuyo contenido se acerca a la noción de perfeccionismo que Huprich et al. (2008) describen como estrategia compensatoria de las personas para evitar el abandono y el rechazo de figuras significativas. A su vez, al buscar correspondencia con la descripción del modelo de rasgos del DSM-5 respecto de la evaluación de la disfuncionalidad cuando se indica la "presencia de disminución de la autoestima y una excesiva autocrítica” (APA, 2013, p. 993).

En el metaanálisis de Nicol et al. (2020) se afirma que un amplio número de esquemas localizados en los diferentes dominios correlacionan con síntomas depresivos, especialmente los que hacen parte del dominio desconexión y rechazo, lo cual es coherente con lo hallado en el presente estudio, donde todos los dominios en que se agrupan los esquemas aportan en la predicción del trastorno depresivo excepto los del dominio de Sobrevigilancia e inhibición. Esto es contrario a lo identificado por Chen et al. (2019), quienes encontraron que este dominio es un buen predictor de la severidad de la depresión al igual que el dominio de desconexión y rechazo. Por otra parte, Davoodi et al. (2018) identificaron que los pacientes depresivos obtienen niveles significativamente mayores en todos los dominios, siendo esto acorde con los resultados obtenidos en el presente estudio, lo cual podría indicar un patrón estable de pensamiento. Esta idea se corrobora con los hallazgos de Flink et al. (2019) en 
relación con la comorbilidad entre trastornos de la personalidad del cluster $\mathrm{C}$ y el trastorno depresivo y la presencia de altas puntuaciones en los esquemas maladaptativos tempranos.

A su vez, en el estudio de Mateos-Pérez y Calvete (2019) se evidenció que los esquemas de desconexión rechazo, junto con las inferencias sociales, fueron predictores de síntomas depresivos en una muestra de adolescentes. Resultado que podría corroborarse en el presente estudio con la muestra clínica adulta, aumentando evidencia sobre el patrón cognitivo presente en pacientes con altos niveles de afectividad negativa.

De acuerdo con la revisión de la literatura realizada se puede concluir que el presente estudio aporta evidencias a favor de la presencia de al menos dos condiciones estables en el funcionamiento afectivo y cognitivo de pacientes con trastorno depresivo: la primera, un alto nivel de afectividad negativa y, la segunda, un patrón cognitivo caracterizado por la presencia de Esquemas maladaptativos tempranos en todos los dominios descritos por Young et al. (2003) (excepto los de sobrevigilancia e inhibición). Esto es congruente con el modelo de rasgos para los trastornos de personalidad descritos en el DSM5, pero además pone el acento sobre la posible identificación de un perfil de personalidad depresivo que puede hacer más vulnerables a las personas al desarrollo de trastornos del estado de ánimo, tal como ya lo evidenciara $\mathrm{Hu}$ prich $(1998,2012,2013)$. En la misma línea, sin embargo, se dejan abiertas preguntas como las descritas por Huprich (2013), respecto de líneas futuras de investigación que den soporte a la formulación de un trastorno depresivo de la personalidad, como serían: a) un estudio más detallado del perfil del trastorno de personalidad depresivo; b) el aporte al desarrollo de un endofenotipo de personalidad depresiva, que pueda apoyarse, por ejemplo, en las evidencias de estudios sobre genética (Örstavik et al., 2012) y electrofisiología (Yu et al., 2019), entre otros; c) el abordaje de la personalidad depresiva en el marco de procesos internos, dinámicas y contenidos que incluya la consideración del sí mismo y la representación de otros, las características de los mecanismos de afrontamiento, las dinámicas motivacionales, las características de la afectividad, la agencia personal y las tendencias de comportamiento (Huprich, 2013). 
Por otra parte, en futuros estudios se podría considerar la adición de un inventario de personalidad que dé mayor soporte a lo aquí presentado. Si bien las medidas empleadas evalúan rasgos estables que pueden considerarse rasgos de personalidad, una medida estandarizada podría dar mayor solidez a los hallazgos. Adicionalmente, sería útil, aumentar el nivel de comparabilidad entre los grupos, ya que, si bien en este estudio la mayoría de las condiciones son similares, hay algunas diferencias en variables como el nivel educativo que podrían modificar los resultados.

En cuanto a las implicaciones clínicas de este estudio resulta relevante enfatizar en la necesidad de incluir el abordaje de los Esquemas maladaptativos tempranos en las terapias actuales para psicopatologías, en especial, la depresión. Así como la consideración de las vulnerabilidades psicológicas en términos de rasgos de la personalidad que pueden predisponer al desarrollo de trastornos emocionales. En cuanto a las implicaciones en prevención y promoción, se destaca el abordaje de la promoción de una infancia saludable, donde las necesidades básicas de los niños sean satisfechas. Debido a que los Esquemas maladaptativos tempranos se generan en edades tempranas, convendría evaluar la necesidad de trabajar sobre el impacto del apego seguro, la autonomía, la libertad de expresión, la espontaneidad y el autocontrol, como condiciones para prevenir el desarrollo de EMT como variables asociadas al desarrollo de psicopatologías.

Finalmente, los hallazgos del presente estudio aportan evidencia a favor de un abordaje dimensional dentro de los trastornos del estado de ánimo que complemente la visión categorial dominante. Adicionalmente, los datos aquí presentados podrían apoyar la discusión sobre la existencia de un trastorno depresivo de la personalidad en la línea en que autores como Huprich y colaboradores lo han planteado y que parece armonizarse con lo descrito en el modelo dimensional de la personalidad en el DSM-5 (2013).

\section{Conclusiones}

Los resultados arrojan evidencia sobre la presencia de altos niveles de afectividad negativa en los sujetos con concurrencia de trastorno depresivo mayor 
y distimia en comparación con sujetos con solo diagnóstico de depresión y con la muestra no clínica, lo cual podría mostrar la presencia de un factor de neuroticismo que podría apoyar la idea de una personalidad depresogénica.

La vulnerabilidad cognitiva expresada en altas puntuaciones en los dominios de desconexión-rechazo, límites inadecuados, perjuicio en autonomía y desempeño y tendencia hacia el otro, estarían indicando, además de coherencia con los resultados de estudios previos, un patrón estable y fijo característico de pacientes con depresión que podría mostrar factores de mantenimiento y compensación del pensamiento de las personas con depresión, coincidente con el modelo de rasgos descrito en el DSM-5. No obstante, a diferencia de los hallazgos de Chen et al. (2019), Davoodi et al. (2018) y Flink et al. (2019) el dominio de sobrevigilancia e inhibición no mostró un valor predictivo en el presente estudio, por lo cual se recomienda para futuras investigaciones controlar el nivel de severidad de los síntomas que podría estar asociado con la presencia de este dominio.

Los resultados apuntan a la presencia de una vulnerabilidad cognitiva y afectiva acorde con el modelo de rasgos de personalidad que describe el DSM-5 que podrían dar soporte a la existencia de un trastorno depresivo de la personalidad.

\section{Referencias}

Achenbach, T. y Edelbrock, C. (1978). The classification of child psychopathology: A review and analysis of empirical efforts. Psychological Bulletin, 85(6), 12751301. doi: 10.1037/0033-2909.85.6.1275

Agudelo, D., Buela-Casal, G., y Spielberger, D. (2007a). Ansiedad y depresión: el problema de la diferenciación a través de los síntomas. Salud Mental, 30(2), $33-41$.

Agudelo, D., Spielberger, D., y Buela-Casal, G. (2007b). La depresión: ̨̇un trastorno dimensional o categorial? Salud Mental, 30(3), 20-28.

Agudelo, D., Gómez, Y. y López, P. (2014). Propiedades Psicométricas del Inventario de Depresión Estado/Rasgo (IDER) con una muestra de población general colombiana. Avances en Psicología Latinoamericana, 32(1), 71-84. 
Relación entre afecto negativo y dominios de esquemas maladaptativos tempranos como variables de personalidad en el Trastorno Depresivo Mayor

Alba, J. y Calvete, E. (2019). Relaciones entre depresión, estrés y esquemas disfuncionales tempranos en adolescentes. Psicología Conductual, 27(2), 183-198.

American Psychiatric Association (2013). Diagnostic and Statistical Manual of Mental Disorders, DSM-5 (5ta. Edición). Washington, D.C.

Bermúdez J. (1978). Análisis Funcional de la Ansiedad. Revista de Psicología General y Aplicada, 153, 617-34.

Besenek, M. (2020). Differences in Dimensional Personality Features in Depressive and Anxious Adolescents. Anatolian Journal of Psychiatry, 21(0), 1. doi: 10.5455/apd.94853

Calvete, E. (2014). Emotional Abuse as a Predictor of Early Maladaptive Schemas in Adolescents: Contributions to the Development of Depressive and Social Anxiety Symptoms. Child Abuse \& Neglect, 38, 735-746.

Cámara, M. y Calvete, E. (2012). Early Maladaptive Schemas as Moderators of the Impact of Stressful Events on Anxiety and Depression in University Students. Journal Psychopathology Behaviour Assessment 34(1) 58-68.

Chen, K., Tam, W. y Chang, K. (2019). Early Maladaptive Schemas, Depression Severity, and Risk Factors for Persistent Depressive Disorder: A Cross-sectional Study. East Asian Archives of Psychiatry, 29(4), 112-117.

Clark, L. y Watson, D. (1991). Tripartite Model of Anxiety and Depression: Psychometric Evidence and Taxonomic Implications. Journal of Abnormal Psychology, 100(3), 316-336. doi: http://dx.doi.org/10.1037/0021-843X.100.3.316.

Clark, L. y Watson, D. (2003). Constructing Validity: Basic Issues in Objective Scale Development. En: Kazdin, A. (Ed.). Methodological issues \& strategies in clinical research (3rd ed., pp. 207-231). New York: American Psychological Association.

Davoodi, E., Wen, A., Dobson, K., Noorbala, A., Mohammadi, A. y Farahmand, Z. (2018). Early Maladaptive Schemas in Depression and Somatization Disorder. Journal of Affective Disorders, 235, 82-89.

Eaton, N.R., South, S.C. y Krueger, R.F. (2010). The meaning of comorbidity among common mental disorders. En Millon, T., Krueger R.F. y Simonsen, E. (Ed.). Contemporary directions ins psychopathology: Scientific foundations of the DSM-V and ICD-11. New York: The Guilford Press.

Ferrando, L., Bobes, J., Gibert, J., Soto, M. y Soto, O. (2000). MINI. Entrevista Neuropsiquiátrica Internacional. Versión en español 5.0.0. DSM-IV. Traducida por L. Franco-Alfonso, L. Franco. 
Flink, N., Honkalampi, K., Lehto, S., Viinamäki, H., Koivumaa冈Honkanen, H., Valkonen囚Korhonen, M. y Lindeman, S. (2019), Early Maladaptive Schemas in Chronically Depressed Patients: A Preliminary Investigation. Clinical Psychology, 23, 15-25. doi: 10.1111/cp.12151

Gotlib, I. y Hammen, C. (2015). Handbook of Depression. Nueva York: Guilford.

Halvorsen, M., Wang, C., Eisemann, M. y Waterloo, K. (2010) Dysfunctional Attitudes and Early Maladaptive Schemas as Predictors of Depression: A 9 Year Follow up Study. Cognitive Therapy Research, 34, 368-379.

Hidalgo, R. y Sheehan, D. (2012). Generalized Anxiety Disorder. In Handbook of Clinical Neurology, 106, 343-362.

Hong, R. y Tan, Y. (2020). Dsm-5 Personality Traits and Cognitive Risks for Depression, Anxiety, and Obsessive-Compulsive Symptoms. Personality and Individual Differences, 169, 110041.

Huprich, S. (1998). Depressive Personality Disorder: Theoretical Issues, Clinical Findings, and Future Research Questions. Clinical Psychology Review, 18, 477500 .

Huprich, S. (2012). Considering the Evidence and Making the Most Empirically Informed Decision About Depressive Personality Disorder in DSM-5. Personality disorders: Theory, Research, and Treatment, 3(4), 470-482.

Huprich, S. (2013). New Directions for an Old Donstruct: Depressive Personality Research in the DSM冈5 Era. Personality and Mental Health, 7(3), 213-222.

Huprich, S., Porcerelli, J., Keaschuk, R., Binienda, J. y Engle, B. (2008). Depressive Personality Disorder, Dysthymia, and Their Relationship to Perfectionism. Depression and Anxiety, 25(3), 207-217.

Klein, D., Schwartz, J., Rose, S. y Leader, J.(2000). Five-year Course and Outcome of Dysthymic Disorder: A Prospective, Naturalistic Follow-up Study. American Journal of Psychiatry, 157(6), 931-939.

Londoño, C. y González-Rodríguez, M. (2016). Prevalencia de depresión y factores asociados en hombres. Acta Colombiana de Psicología, 19(2), 315-329.

Londoño, N., Calvete, E., Ferrer, A., Chaves, L. Castrillón, D., Schnitter, M. Marín, C. y Maestre, K. (2012). Young Schema Questionnaire-Short Form: validación en Colombia. Universitas Psychologica, 11(1), 147-164.

Mateos-Pérez, E. y Calvete, E. (2019). Interacción entre diferentes factores cognitivos en la predicción de los síntomas de depresión. Behavioral Psychology, 27, 21-39. 
Relación entre afecto negativo y dominios de esquemas maladaptativos tempranos como variables de personalidad en el Trastorno Depresivo Mayor

Mineka, S., Watson, D. y Clark, L. (1998). Comorbidity of Anxiety and Unipolar Mood Disorders. Annual Review of Psychology, 49, 377-412.

Nia, M., Sovani, A. y Forooshani, G. (2014). Exploring Correlation Between Perceived Parenting Styles, Early Maladaptive Schemas, and Depression Among Women With Depressive Symptoms in Iran and India Role of Early Maladaptive Schemas as Mediators and Moderatos. Iranian Red Crescent Medical Journal 16(12), e17492.

Nicol, A., Mak, A., Murray, K., Walker, I. y Buckmaster, D. (2020). The Relationships Between Early Maladaptive Schemas and Youth Mental Health: A Systematic Review. Cognitive Therapy and Research, 44, 715-751.

Örstavik, R., Kendler, K.., Czajkowski, N., Tambs, K. y Reichborn-Kjennerud, T. (2007). The Relationship Between Depressive Personality Disorder and Major Depressive Disorder: A Population-based Twin Study. The American Journal of Psychiatry, 164, 1866-1872.

Rafi, M., Adibsereshki, N. y Aval, M. (2017). The Mediating Role of Early Maladaptive Schemas in the Relationships of Emotional Maltreatment and Emotional Disorders. European Journal of Mental Health, 12(1), 104-115. doi: 10.5708/ ejmh.12.2017.1.7.

Renner, F., Lobbestael, J., Peeters, F., Arntz, A. y Huibers, M. (2012). Early Maladaptive Schemas in Depressed Patients: Stability and Relation with Depressive Symptoms Over the Course of Treatment. Journal of Affective Disorders, 136(3), 581-590. doi: 10.1016/j.jad. 2011.10.027.

Rhebergen, D. y Graham, R. (2014). The Re-Labelling of Dysthymic Disorder to Persistent Depressive Disorder in DSM-5: Old Wine in New Bottles? Current Opinion in Psychiatry, 27(1), 27-31.

Ritterband, L. y Spielberger, C. (1996). Construct Validity of the Beck Depression Inventory as a Measure of State and Trait Depression in Nonclinical Populations. Depression and Stress, 2, 123-145.

RStudio Team. (2020). RStudio: Integrated development for R. RStudio, Inc., Boston MA. RStudio

Sheehan, D., Lecrubier, Y., Sheehan, K., Amorim, P., Janavs, J., Weiller, E., Hergueta, T., Baker, R. y Dunbar, G. (1998). The Mini-International Neuropsychiatric Interview (M.I.N.I): The Development and Validation of a Structured Diagnostic Psychiatric Interview for DSM-IV and ICD-10. The Journal of Clinical Psychiatry, 59(20), 22-33. 
Spielberger C., Buela-Casal, G. y Agudelo, D. (2008). Inventario de depresión estado-rasgo: Manual. Madrid: TEA ediciones.

Spielberger, C., Gorsuch, R. y Lushene, R. (1970). Manual for the State-Trait Anxiety Inventory. Palo Alto, California: Consulting Psychologist Press.

Spielberger, C. (1983). Manual for the State-Trait Anxiety Inventory: STAI (Form Y). Palo Alto, CA: Consulting Psychologists Press.

Spielberger, C. (1988). Manual for the State-Trait Anger Expression Inventory (STAXI). Florida: Psychological Assessment Resources, Inc.

Spielberger, C., Agudelo, D., Carretero-Dios, H., De los Santos-Roig, M. y Buela-Casal, G. (2004). Análisis de ítems de la versión experimental castellana del Cuestionario de Depresión Estado-Rasgo (ST-DeP). Análisis y Modificación de Conducta, 30, 495-535.

Spielberger, C., Jacobs, G., Russell, S. y Crane, R. (1983). Assessment of Anger: The State-Trait Anger Scale. En: Butcher, J. y Spielberger, C. (Ed). Advances in Personality Assessment, 2, 161-189.

Spielberger, C., Ritterband, L., Reheiser, E. y Brunner, T. (2003). The Nature and Measurement of Depression. Revista Internacional de Psicología Clinica y de la Salud/International Journal of Clinical and Health Psychology, 3, 209-234.

Spielberger, C., Ritterband, L., Sydeman, S., Reheiser, E. y Unger, K. (1995). Assessment of Emotional States and Personality Traits: Psychological Vital Signs. En: Butener, J. (Ed.). Clinical Personality Assessment: Practical Approach (pp. 42-58). New York: Oxford University Press.

Testal, J., Calderón, C. y Garcelán, S. (2014). Del DSM-IV-TR al DSM-5: análisis de algunos cambios. International Journal of Clinical and Health Psychology, 14(3), 221-231.

Tyrer, P., Seivewright, H. y Johnson, T. (2004). The Nottingham Study of Neurotic Disorder: Predictors of 12-year Outcome of Dysthymic, Panic and Generalized Anxiety. Psychological Medicine, 34(8), 1385-1394.

Watson, D., Clark, L. y Carey, G. (1988). Positive and Negative Affectivity and Their Relation to Anxiety and Depressive Disorders. Journal of abnormal psychology, 97(3), 346-353.

Willner, C., Gatzke-Kopp, L. y Bray, B. (2016). The Dynamics of Internalizing and Externalizing Comorbidity Across the Early School Years. Development and Psychopathology, 28(4-1), 1033-1052. 
Relación entre afecto negativo y dominios de esquemas maladaptativos tempranos como variables de personalidad en el Trastorno Depresivo Mayor

Young, J., Klosko, J. y Weishaar, M. (2003). Schema Therapy: A Practitioner's Guide. Guilford Press.

Yu, H., Gu, S., Yao, F., Wang, Z. y Fu, W. (2019). Electrophysiological Characteristics in Depressive Personality Disorder: An Event-Related Potential Study. Frontiers in Psychology, 9, 2711. 